Original Research Paper

\title{
Mr. Dr. Health-Assistant Chatbot
}

\section{Md Meem Hossain ${ }^{1}$, Salini Krishna Pillai ${ }^{1}$, Sholestica Elmie Dansy ${ }^{1}$, Aldrin Aran Bilong ${ }^{1}$, Ismail Yusuf Panessai ${ }^{1}$}

${ }^{1}$ Department of Computing, Faculty of Arts, Computing Industry Creative. Universiti Pendidikan Sultan Idris. Perak, Malaysia.

Article History

Received:

30.10 .2021

Revised:

17.12.2021

Accepted:

22.12.2021

*Corresponding Author:

Md Meem Hossain

Email

mdmeemhossain@gmail.com

This is an open access article, licensed under: $\mathrm{CC}-\mathrm{BY}-\mathrm{SA}$
Abstract: Research says $60 \%$ of visits to a doctor are for simple small-scale diseases, $80 \%$ of which can be diagnosed at home using simple check-up. These diseases mostly include common cold and cough, headache, abdominal pains etc. Whereas, chat-bots in healthcare are highly in demand, which functioning can offer various services from symptom checking and appointment scheduling. Therefore, the purpose of the research aims to design, develop and evaluate a health-assistant Chat-bot Application entitled "MR.Dr." that helps users to ask any personal query related to healthcare without physically available to the hospital. MR.Dr. is evaluated in term of usability. 30 respondents attended the survey of usability evaluation. In the system usability scale MR.Dr. achieved $87.6 \%$ rating which means Grade A (excellent). User's feedback level was pretty satisfying where $24 / 7$ service is the highest one.

Keywords: Chatbot, e-Health, Natural Language Processing. 


\section{Introduction}

Machines have started to impersonate as human with the advent of artificial intelligence, machine learning, and deep learning. Artificial intelligence is now at its peak and chatbot is just example of its to contribute to progress [1]. Chatbot are entering our every lives, such as Amazon, Alexa and Google Assistant. Chatbots are also used in applications such as e-commerce customer service, call centers and internet games. With a chatbot, one can communicate with a text or voice interface and get response through artificial intelligence [2]. ELIZA was interestingly one of the first chat-bots developed in 1966, which happened to be a psychotherapist. It was just a computer program designed to answer user queries like a psychology professional to an extent where user believed that it is talking to real doctor. Health-care sector is no different. Chatbots are slowly being adopt in health-care. The global chatbot market is estimated to teach at least $\$ 1.23$ billion by 2025 [3].

Chatbots as part of AI devices, are Natural Language Processing (NLP) systems acting as a virtual conversational agent mimicking human interactions [4]. So, this is a platform are focused on Artificial Intelligence and represent machine that answers questions using Natural Language processing (NLP).

Artificial Intelligence (AI) is an umbrella term for computer science consisting of a complex mathematical algorithm that process input information to produce any specific predefined outputs which lead to relevant outcomes [5].

Artificial intelligence systems, which utilize large data set, can be designed to enhance decision making and analytical process while imitating human cognitive function. AI has been applied in medicine and various health-care services such as diagnostic, as well as clinical laboratory, screening and health communication [6] [7].

Natural Language Processing (NLP) is used in making of this chatbot with is an important component of artificial, so we can imbibe same thing in our chatbot for generation of accurate and responsive answers [8]. Artificial intelligence makes it possible for the chatbot to analyze the conversation and NLP helps to interpret the text.

Chatbots functioning depends on Natural Language Processing (NLP) that helps users to submit their problems about the health. The main purpose of the chatbot to build the language gap between the user and health providers by giving immediate replies to the Questions asked by the user. Today's people are not concern about their personal health. They avoid to go in hospital for small problem which may become a major disease in future [9]. A big disease can start from small problems such as head ach which facts normal but it may beg of big disease such as brain tumor. Most of diseases can be identified by common symptoms so, the diseases can be predicted if the patient body is analyzed periodically [8].

Many of the existing system have some limitation such as there is no instant response given to the patients, they have to wait for the expert's acknowledgement for a long time. Some of the process may change amount to perform live chat or telephony communication with doctors online [10]. Chatbots in health-care are highly in demand, medical institution can offer various services from symptoms checking and appointment scheduling to deal with additional questions. People are able to get answers to their questions with the help of chatbot. There is no need for them to call the clinic to clarify some misunderstanding.

Since the discovery of the corona virus (Covid-19), it has become a global pandemic, at the same time, it has been a great challange to hospitals or health-care staff to manage the flow of the serious pandemic like novel corona virus (covid-19), chatbots are beneficial as a supplement to personal clinical case or immediate medication (Batteneni, 2020). There are some eshtablished covid-19 virtual agents integrated with messenger application. The World health Organization (WHO) has launched a dedicated messenger app in seven languages to keep the public safe from corona virus infections (World Health Organization (WHO), 2020).

Normally Users are not aware about all the treatment or symptoms regarding the particular diseases. For small problem users have to go to personally to the hospitals for check-up which is more time consuming also handling the telephone calls fro the complaints is quite hectic. Such a problem can be solved by using medical chatbot by giving proper guidance regarding healthy living [9]. Chatbot also could help doctors, nurses, patients on their families. Better Organization of patient information medications management helping in emergencies or with first aid, offering a solution for superficial medical issues; these are all possible situation for chatbots to step in and reduce the burden on medical professionals [11]. 


\section{Literature Review}

\subsection{Chatbot}

Chatbot is an artificially intelligent creature which can converse with human. This could be text-based or a spoken conversation (in case of voice-based queries). Chatbots are basically used for information acquisition. It can run on the local PCs and Mobile Phones, though most of the time it is accessed through internet [12]. Chatbots, as part of Artificial Intelligence devices are Natural Language processing (NLP) systems acting as a virtual Conversational agent impersonate human interaction [4]. While this technology is still in its development stage. Health chatbots could potentially increase to health-care, improve doctor-patient and clinic-patient communication or help to manage the increasing demand for health services such as via remote testing, medication adherence, observing or tale-consultation [13].

\subsubsection{Categories of Chatbot}

Categories of Chatbot are [14]:

1. Goal-based Chatbot

Goal-based chatbots are for achieving the primary goal aim. They are programmed for doing the particular task and setup to have small conversations to get information from the user to complete their task. For example, an organization can deploy a chatbot on their websites to help their customers to answer their questions.

2. Knowledge-based Chatbot

Knowledge-based chatbots are classified based on the knowledge they access from the data sources or data they are trained on. There are two main data sources - open domain and closed-domain. Open-domain are for responding for general topics. For example, opendomain are Quiz Bowl, News, and Sports. Closed-domain are for answering particular knowledge domain. All data required for answering questions is provided in the data set such as Daily Mail etc.

3. Service-based Chatbot

Service-based chatbots are for providing services to the customer. It can be for personal or commercial purpose. For example, Courier Company can provide copies of delivery documents through a chatbot instead of phone calls or we can make order cloths from Macys.

4. Response generated Chatbot

Response Generated-based chatbots are classified as per the action they take in response generation. For response models input and output is in natural language text. The dialogue manager is responsible for integrating response models together. To get an appropriate response, dialogue manager follows three steps. First, it uses all response models to generate a set of responses. Second, returns a response based on priority. Third, if no priority response, the response is selected by the model selection policy.

\subsubsection{Understanding Language and Context}

The chatbot tries to mimic different capabilities and functions of human brain. Understanding Language and Context includes:

- Reinforcement Learning:

It is the ability to accept rectifications over time to improve suitability of responses. It allows the Chatbot to improve and learn more from the user inputs.

- Machine Learning:

It is the method of data analysis which automates analytical model building by learning from data, patterns and make the decision appropriately without human intervention. It learns from how to respond to the user by analyzing the previously given responses. This capability is very important for qualitative intent recognition.

- Intent Recognition:

It is the ability to guess what user is requesting for. This is necessary to setup the natural conversation as it reduces user's frustration. It also enables virtual agent to start a relevant conversation with human.

- Natural Language Processing:

It is the ability to read the text and understand natural sentence structure and simple keywords also known as Triggers. Entity Recognition: It helps to understand that some of the keywords 
refers to informative categories. For example: "May 19th" is a date. This is required for more complex commands and analysis.

- Dialog Management:

Chatbot follows conversations, maintains, recall and memorize conversation. This is important for starting natural, human-like conversation.

- Q/A Pairs / Scripts:

It is used to deliver facts, solutions to queries or questions. Chatbot gets these facts from good Intent Recognition [15].

\subsubsection{Human Computer Speech Interaction}

Speech recognition is one of the most natural and sought-after techniques in computer and networked device interaction has only recently become possible (last two decades) with the advent of fast computing.

Speech is a sophisticated signal and happens at different levels: "semantic, linguistic, articulatory, and acoustic" [16]. Speech is considered as the most natural among the aspects of human communication, owing to copious information implicitly existing beyond the meaning of the spoken words. One of the speech information extraction stages is converting speech to text via Automatic Speech Recognition (ASR) a mining speech information [17]; then, the resulting text can be treated to extract the meaning of the words.

Speech recognition is widely accepted as the future of interaction with computers and mobile applications; there is no need to use traditional input devices such as the mouse, keyboard or touch sensitive screen and is especially useful for users who do not have the ability to use these traditional devices [18]. It can help disabled people with paralysis, for example, to interact with modern devices easily by voice only without moving their hands.

\subsection{Chatbot as e-Health in Health-Care}

Chatbots are becoming a necesity in several industries. Gather plans that more than $85 \%$ customer interctions will be changed without human by 2020 [19]. Chatbots are expected to be the number one consumer application of AI over the next five years according to technology [20]. Healthcare chatbot is a system designed to improve the e-health paradigm by using a chatbot to simulate human interaction in medical contexts. A chatbot can be successfully designed to work as a helping tool in doctor-patients communication, but it must be emphasized that it should work as supplement and never replacement. All healthcare providers are always willing to help their patients and they understand how it is vital to be available if there is urgent need of medical attention. Unfortunately, doctors have limited time and a lot of patients which does not allow to be available anytime. In the turn, chatbots are there for those who need medical assistance at all time. Furthermore, virtual assistants may be responsible for remembering users to take their medicine and monitoring a patient's health status, Abbreviations and Acronyms [21].

As a consequence, health and fitness chatbots have begun to gain the popularity in the market. Previous year Facebook has started allowing healthcare industries to create messenger chatbots which would communicate with users. A great example is Health Tap the first company to release a health bot on the messenger app. It allows users to ask their medical related queries and receive answers [22]. Chatbot has been the recent advances of technologies for data processing and analytic have radically charged the healthcare giving rise to digital healthcare solutions promising to transform the whole healthcare process to become more efficient, less expensive and higher quality [23]. Technology-based self-services channels and digital health interventions have the potential to support patients in their everyday life and health professionals.

As chatbots in healthcare are highly in demand medical institutions can offer various services from symptom checking and appointment scheduling to dealing with additional questions. People are able to get answers to their additional questions with the help of chatbot. There is no need for them to call the clinic to clarify some misunderstanding.

\subsection{Artificial Intelligence}

Before few years, a computer could hardly think as the human brain do. But today, AI has changed everything we can now solve complicated problems easily. The fundamental technologies for chatbot are machine learning, natural language processing (NLP), and Artificial intelligence (AI). These 
technologies brought chatbot invention hereafter brands communication, to a completely new personalized level. Although chatbot solutions for business are mostly used in the industry of customer service, the technical giants such as IBM, Google, and Microsoft suggest that the true potential of chatbots still needs to be fully revealed. AI provides many opportunities, as it includes such capabilities that allow the software to perform such tasks that humans perform. Natural language processing is the foundation of AI based chatbots. By using sophisticated algorithms of NLP, chatbots can process the input text: understand, conclude, and determine that what was said or written and then state a list of all suitable actions. If there was no development in the field of NLP, chatbots had to be at the same and awkward situation as they were at their beginning [11].

Artificial Intelligence can be used to predict any disease and to provide the list of possible treatments based on given symptoms. Moreover, if periodically analysis is performed on a person's body, AI can help to predict any possible disease even before any damage occurs to the body. The significant challenges for this study are research and development cost and support from the government for the proper successful implementation of all medicines, which are not described in this research paper [8]

Current wave of research has taken up the challenge of developing intelligent chatbots able to answer users' questions utilizing AI. The concept of the chatbot has gained increasing attention in the last few years [24]. As stated by Sundar Pichai, Google CEO, "We are now witnessing a new shift in computing the move from a mobile-first to an AI-first world".

\subsection{Natural Language Processing}

Natural Language Processing NLP is a subcategory of Artificial Intelligence can come in. NLP based chatbots as of now have the abilities of well and really imitating human conduct and executing a horde of errands. With regards to actualizing the equivalent on a lot bigger use case, similar to a clinic - it tends to be utilized to parse data and concentrate basic strings of information, accordingly offering an open door for us to use unstructured information [25]. Hudaa et al. [25] showed how Natural Language Process (NLP) works First level until health-care chatbot.

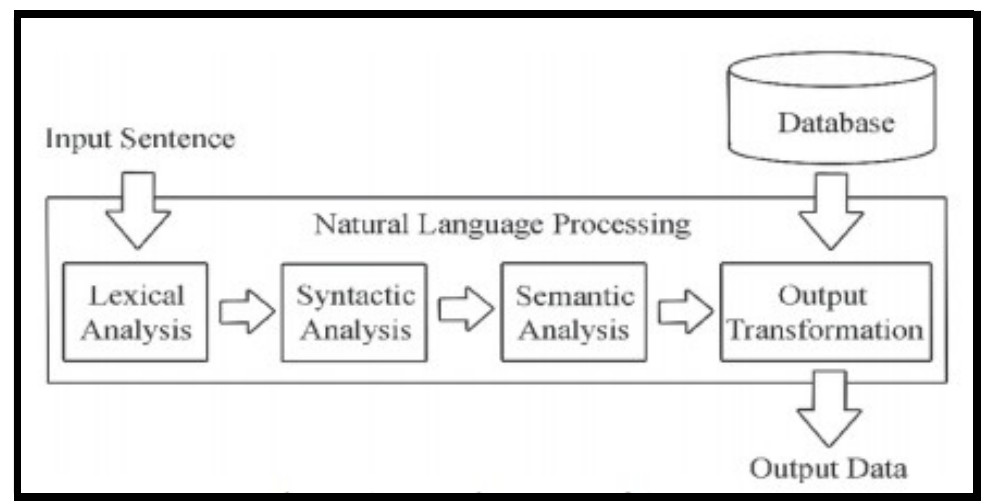

Figure 1. NLP is a Core of AI

Figure 1 (Level 1) explains that NLP is a control of software engineering that requires aptitudes in man-made consciousness, computational semantics, and other AI disciplines.

Next, Figure 2 (Level 2) shows numerous natural language handling frameworks "learn" after some time, reabsorbing the aftereffects of past connections as input about which results were precise and which did not meet desires. These AI projects can work dependent on likelihood, which gauge the probability that a given bit of information is really what the client has mentioned. In view of whether that answer meets endorsement, the probabilities can be balanced later on to meet the developing needs of the end-client.

Last level Figure 3 intend to explain NLP, a part of AI, goes for fundamentally decreasing the separation between the abilities of a human and a machine. As it getting increasingly more footing in the medicinal services space, providers are concentrating on creating arrangements that can comprehend, break down, and produce languages would humans be able to can get it. 


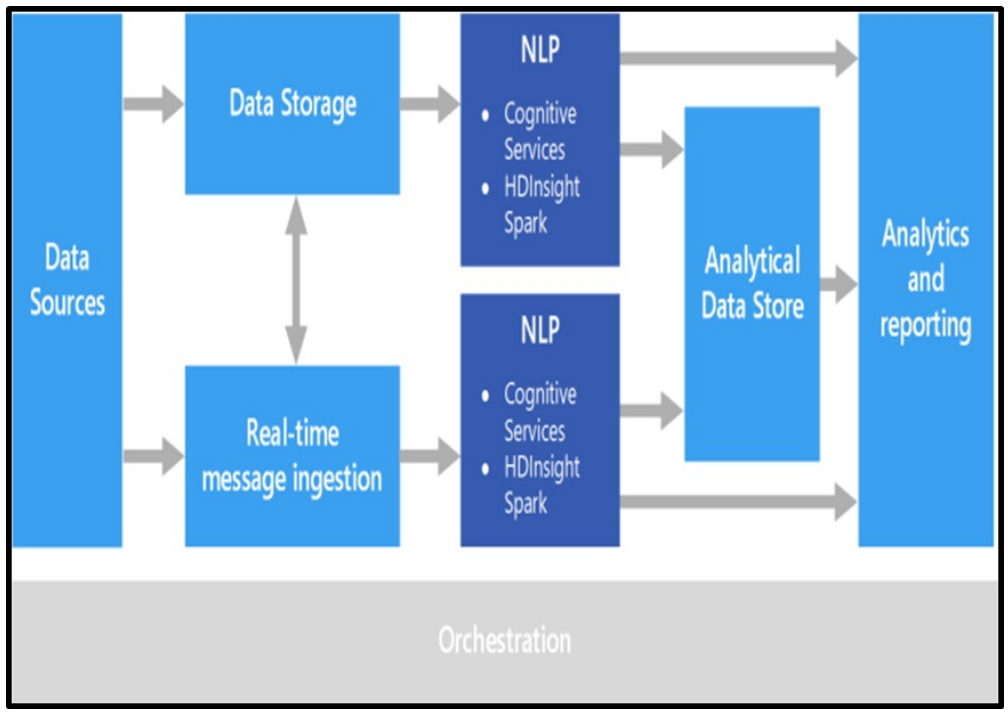

Figure 2. Natural Language Processing

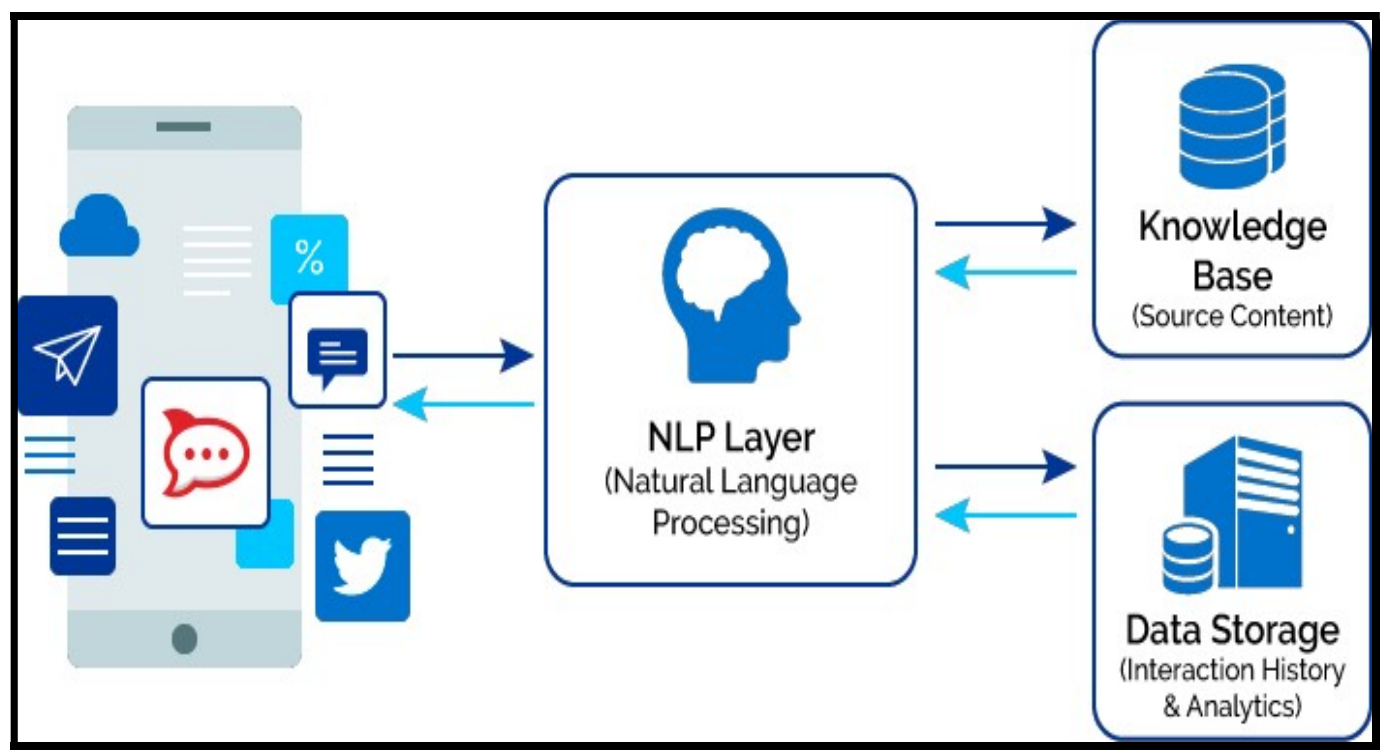

Figure 3. NLP in Health Care

NLP Natural Language Processing acts as a fundamental pillar for recognition of language, which is used by Apple's Siri and Google. It allows technology to recognize human natural language text and speech-based commands and include two major components natural language generation (NLG) and natural language understanding (NLU). Natural language understanding is more laborious than natural language generation, as the natural language has a remarkably rich structure and form. It maps the given input and analyzes multiple features of the language [11].

NLU is responsible for handling and converting formless data into a proper form that the system can easily understand [2]. While, NLG involves text realization and text planning to generate an understandable response. In simple words, language generation is responsible for the formation of linguistically correct sentences and phrases. The key challenge faced by NLP is to understand the complications of natural human language 
The structure of language is itself very vague regarding syntax, lexis, and other components of speech such as similes and metaphors. A single word can be taken as a noun or a verb; a single sentence can be passed in many different ways; moreover, a single input may have multiple meanings, etc.

A fundamental component understands what any user says at a given time and then converts this language into well-defined input that can be further processed by the system. As chatbots are domainspecific so they should support multiple features. The natural language processing engine consists of the latest algorithms of machine learning that are used to identify the intent of the user and then match them with the list of those intents that are supported by the bots (Higoshinaka, Miyazaki, sugiyama and matsuo, 2014).

\subsection{Python Programming Language}

AI is a large field of study and helps find solutions to real-world problems. Fields of study in AI are machine learning, logic, searching, artificial neural networks, genetic algorithm, and knowledge representation. AI is applied in gaming, natural language processing, expert systems, vision systems, speech recognition, handwriting recognition, intelligent robots [26]. Python is an example of a language that does everything right within the domain of things for which it is designed. What sets Python apart is that the application can be written on one platform and can be used on other platforms. Python code is readable and has a concise syntax that allows applications to be written using fewer lines of code than other programming languages. Python supports functional, imperative, objectoriented, and procedural coding styles. Python provided for educational and other purposes for which other programming languages may fail [27].

There are various programming languages such as Lisp, Prolog, $\mathrm{C}++$, Java, and Python, which can be used to develop AI applications. Among them, the Python programming language is gaining in popularity, and some of the reasons are that Python includes very little coding and simple syntax among other programming languages that can be developed for AI application development. Testing for this reason can be easier and can focus more on programming itself. The main advantage of using Python for AI is that it comes with built-in libraries. Python has libraries for almost all types of AI projects. For example, NumPy, SciPy, matplotlib, nltk, SimpleAI are some of the important embedded Python libraries [28].

Python is a programming language that has English-like syntax. It makes it easier to write, read and understand code. Python is a very productive language, because due to its simplicity, the focus is on solving problems. Python is an interpreted language which means it is executed directly code by line. In the event of any error, execution is stopped and the error that occurred is reported. Python shows only one error, even if there are multiple errors. This makes it easier to troubleshoot. Python does not know the type of variable until the code is run. When executed, it automatically assigns a data type. The programmer does not have to worry about declaring variables and their data types. Python comes under an open-source license approved by OSI, which makes it free to use and distribute. The source code can be downloaded, modified, and distributed as own version of Python. Python's standard library is huge, and there are almost all functions for solving various tasks. Python package manager (pip) facilitates the import of other packages from the Python package index (PyPi). Python code can run on different platforms [29].

\section{Methodology}

\subsection{Research Method}

Rapid Application Development or RAD used in this research. RAD is one type SDLC model. It is a model that is suitable for small project which need to be finish on short period of time. Mobile Application on L-CPR is about creating a mobile application for teaching CPR. It is not considered as a large project as it complexity is not that high. Besides that, this project also does not require some expensive and high technology gadget which will cause a high risk in the project.

RAD is a better development model as compared to the traditional SDLC model. The traditional SDLC model follows a rigid process model which more emphasize on requirement analysis and gathering before the coding process start. The customer has to make a confirm requirement which usually cannot be change at later time. Due to this, user will not get the feel of the products as there is no working prototype for a long period of time ("SDLC - RAD Model"). In term of this problem, $\mathrm{RAD}$ is more flexible. There will be a prototype being built to allow user see the progress of the 
project.Sometime user might feel that their original requirement is not suitable after seeing the prototype. In this case, they can give feedback to developer. Developer can go back to the design phase all update any requirement in the design phase of RAD. This make RAD is flexible as it can always swap between the construction phase and design phase in order to satisfy user requirement.

Furthermore, the development time in RAD is faster. This is due to the prototype is being created during the project development. All the prototype that being built during the project development is kept for future use. If a component is being picked from previous prototype and it is tested before, the testing time can be saved as it no need to be tested for second time. Hence, the re-usability of the component can help in reduce the development time. In relation to this, the cost for development will also reduce.

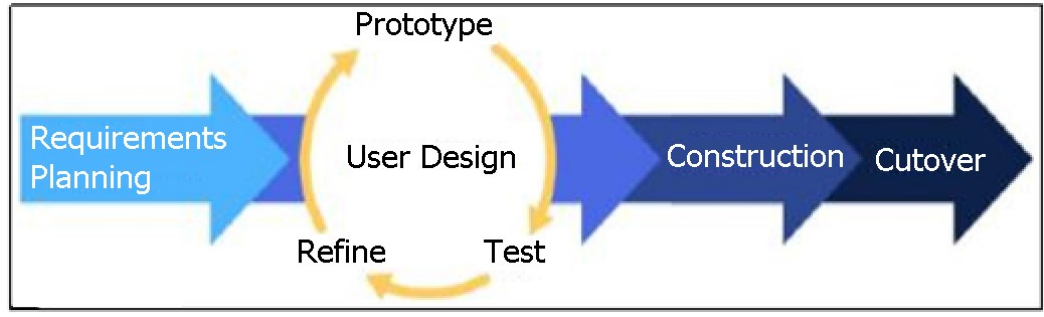

Figure 4. Phase in SDLC

The RAD planning phase is initial process to gather the specific requirement of the system that will develop. In additional, the phase defines the business function and data subject areas that the system's scope. Planning is the key of successful a system development. So, this phase should be conducted properly.

During design phase, analyst convert the description of the alternative solution into logical and then physical system specification. Complete the analysis by creating action diagrams defining the interactions between process and data. System procedures are designed and preliminary layouts of screens are developing. The programmers are ready to be return over for construction phase.

In the development phase, the code will be generated as well as database descriptions for the final product. The design that created during the design phases added. The last phase is cut-over phase. When the cut-over phase occurs, a variety of actions are needed, comprehensive testing, training of the end-users, organizational changes and the operation parallel with the previous system until new system.

\subsubsection{Requirement Planning}

The objectives of the Requirement Planning stage are:

- to establish a general understanding of the business problems that surround its development and eventual operation

- to become familiar with existing systems, and

- to identify the business processes that will be supported by the proposed application.

The RP stage begins with a meeting or series of meetings, between the businesses and IS management. These meetings initiate the development process by establishing a mutual understanding of the objectives of the development project in general and the system in particular.

Business executives, end users and IS professionals, then, take part in Joint Requirements Planning (JRP) workshops which progress through a structured set of steps. During this stage, an outline of the system area and definition of the system scope are developed.

The tasks of this stage are:

- Research Current Situation

This task initiates the RP stage for the proposed system by researching the current environment. The information developed in the initial discussions provides a starting point for this investigation. This research is conducted for preparing the requirements definition through JRP workshops. The project team members become familiar with the current situation 
by investigating current systems, the information available in any existing CASE repositories, and by looking at similar systems for the purpose of looking for any reusable structures.

- Define Requirements

The outline system area model and scope of the proposed system are developed in this task. The functionality of the system is expressed in terms of the business processes and the data that the system will support. Management issues that affect subsequent development and transition activities are also identified. Deliverables of this task are accomplished via a series of JRP workshops. Participants in these workshops include the managers of business areas that will be directly affected by the operation of the proposed system.

- Finalize Requirements

In this task, the scope of the proposed system is formally documented. An estimate of the cost and duration to implement the system is prepared. Sometimes, the cost and duration may already be fixed when the project commences. In this case, the scope must be well defined such that the project is still viable with the cost and duration provided. Approval to proceed with the implementation is then obtained.

\subsubsection{User Design}

The objectives of the UD stage are:

- to analyze in detail business activities associated with the proposed system area

- to analyze in detail the business data associated with the proposed system area

- to develop the system structure in terms of the automated and manual functions that will comprise the system

- to develop proposed screen layouts for the most important automated functions

- to select the appropriate construction approach for the system, and

- to prepare a work plan defining the steps necessary for transition of the system, the effort required to perform these steps, and a schedule by which these steps can be completed.

The UD stage produces a detailed system area model, an outline system design, and an implementation plan. End users participating in Joint Application Design (JAD) workshops perform the analysis and design activities associated with this stage.

The tasks of this stage are:

- Produce Detailed System Area Model

JAD workshops are conducted to complete the analysis of the business activities and data associated with the proposed system, and produce a detailed system area model. Once the detailed system area model is completed, it is possible to refine the scope to ensure that the critical functions will be delivered in the required time frame.

- Develop Outline System Design

An outline system design is developed by the JAD workshop participants using the system area model that has been completed and confirmed. Upon completion of the outline design, interactions between procedures (series of functions) and data are identified. That is, data usage per function is defined. The deliverables produced in this task include functions required for the system, reusable design components to be incorporated into the system design, system structures and tentative layouts of critical screens and reports to be supported by the system.

- Refine System Design

The design that emerges from the JAD workshops is reviewed by the project team members and the user design team. The consistency of the analysis and design is confirmed via interaction analysis and prototyping. By interaction analysis, the interactions between functions and data are analyzed to identify missing/extraneous functions (no data usage) or data (not used by any function). The completeness of the detailed system area model and outline system design is verified. When inconsistencies are resolved, prototypes of screens are developed and shown to the users for review and revision. Adjustment to the list of open issues is then made.

- Prepare Implementation Strategies

A plan for the implementation of the system is developed. The implementation approach is selected after reviewing the design of the system. To speed up delivery, parallel development 
and time box development can be adopted. An implementation plan is prepared listing all the tasks that must be performed to develop the system and convert it into operational use. An estimate of the effort necessary to complete each task is made and summarized into an overall project cost estimate.

- Finalize System Design

The outline design of the system and implementation plan needs to be finalized. Usually, JAD workshops are conducted. Workshop participants suggest changes to the design based on their experience in reviewing the prototypes and further research into the system requirements. Open design issues are discussed and resolved except those with no impact to the system design such as cultural issues. The implementation plan for the system is presented and discussed, enabling workshop participants to point out any unforeseen problems or invalid assumptions in the plan.

- Obtain Approval for Construction

The results of the final JAD workshop are incorporated into the system design and implementation plan, and approval to proceed to the Rapid Construction Stage is sought.

\subsubsection{Construction}

The objectives of the Construction stage are:

- to complete the detailed design of the proposed system

- to create and test the software that implements the proposed system

- to generate a system that operates at an acceptable level of performance

- to prepare documentation necessary to operate the proposed application

- to design, develop, and test the required transition software, and

- to perform the steps necessary to prepare for the conversion of the system to production status.

The design of the proposed system, initially described in the UD stage, is completed in the RC stage, and application software to implement that design is developed and tested. Activities to prepare for the transition of the system to production status are also performed.

The tasks of the Construction stage are:

- Prepare for Construction

The development environment is finalized including workstations and workspace for the system developers, and the CASE software for their use. Additionally, the database is designed based on the preliminary data structure developed in the UD stage. Finally, the testing strategy for the system is completed and the acquisition of facilities necessary to operate the system after it is constructed is initiated.

- Construct System

The detailed definition of the design of each function is completed, based on the requirements of the future end-users. Software to implement the automated functions is developed and tested.

- Generate Test Data and System Documents

This task involves developing the necessary test data that will verify the operational capacity of the system. This data will be used during the integration, system and acceptance tests. Documentation explaining how the system is to be operated by users and computer operations personnel is produced.

- Prepare for Cut-over

Activities in this task are to prepare for the subsequent conversion of the application into production status. These activities include:

$\circ$ to prepare a detailed work plan for transition activities and a contingency plan to cover failure of the converted application;

○ to develop software needed to convert data from existing formats into formats used by the application;

○ to develop transition procedures and arrangements;

$\circ$ to prepare a user training plan and training materials; and

○ to resolve organizational issues related to deployment of the new application. 
- Verify System Construction

The system must be put through a series of tests to ensure that each component of the system and the complete system perform according to the user requirements.

\subsubsection{Cut-Over}

The objectives of the TR stage are:

- to install the system in production operation with minimal disruption of normal business activity

- to maximize the effectiveness of the system in supporting the intended business activities, and

- to identify potential future enhancement.

The system developed in the construction stage becomes operational in the cut-over stage. At this time, the developers prepare existing data for the new system and train users to operate the new application. They also provide support to resolve any problems that arise immediately after the application becomes operational.

The tasks of the Cut-over stage are:

- Conduct User Training Training sessions are conducted to instruct future users of the new system on how it operates. This training is completed before the application is placed into production. The training sessions are based on materials developed during the $\mathrm{RC}$ stage and follow the training plan that was developed at that time. The training may include a train-the-trainers program if a large number of users have to be trained or permanent training is required.

- Perform Data Conversion Information necessary for the operation of the new system is converted from existing data sources into a format accessible by the new system. The converted data is then loaded into the data structures associated with the system.

- Install Production System

Steps necessary to commence operation of the system in the production environment are performed in this task. Necessary adjustments to the hardware and system software configuration are completed, instructions given to the operations personnel who will be operating the system, and software libraries loaded with the production versions of the application software.

- Accept System Installation

The new system installation can be regarded as successful and will be accepted when it operates for a specified period within defined tolerances for performance, error rate and usability. This acceptance is based upon agreements among users, production/operations staff, support staff, and the information management organization about schedules, procedures, pricing policies, warranty agreements, software, and systems documentation.

\subsection{Research Instrument}

The development of instrument was based on close ended questionnaires to gathering the information of Health Assistant Chatbot application. Basically, the purpose of the questionnaires is to determine the aspect of Health Assistant Chatbot application in terms of usability and functionality by collecting their feedback for the analysis purpose.

Different questions will been construct to be answered by students. This instrument was measured using the Likert Scale from 1 (poor) - 5 (most liked). The questionnaire were distributed to 15 respondents from Sultan Idris Education University who always used to go to the UPSI clinic for their health issues.

\subsection{System Architecture}

System Architecture shows how system work. Such phone stores related application includes mobile API, and to store Firebase database required. We used tier concept include three tier such front tier, middle tier and back tier. Front tier is what user can see which is User interface, middle tier is progress of verification, validations and function which unable seen by user. Also consider as intermediate person helps to ease progress. Back tier is store and retrieve data. 


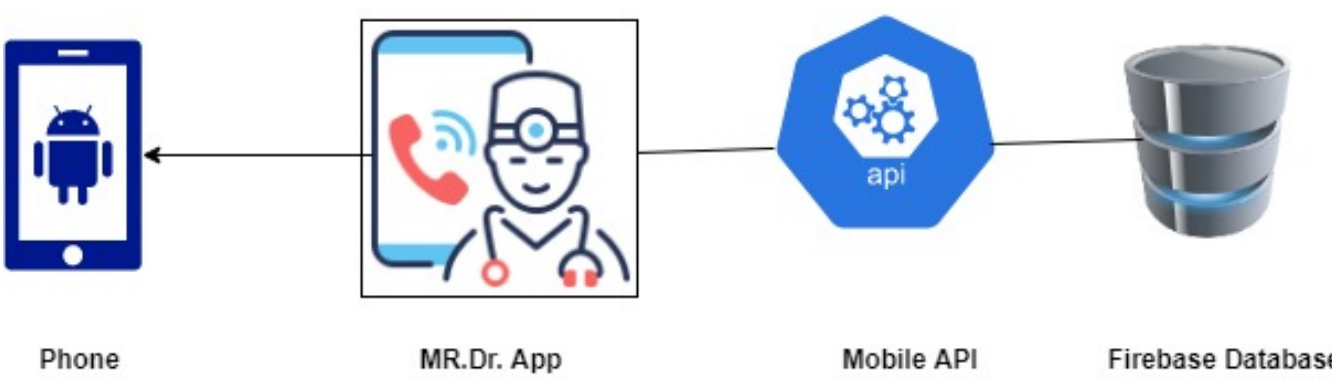

Figure 5. First Level of System Architecture

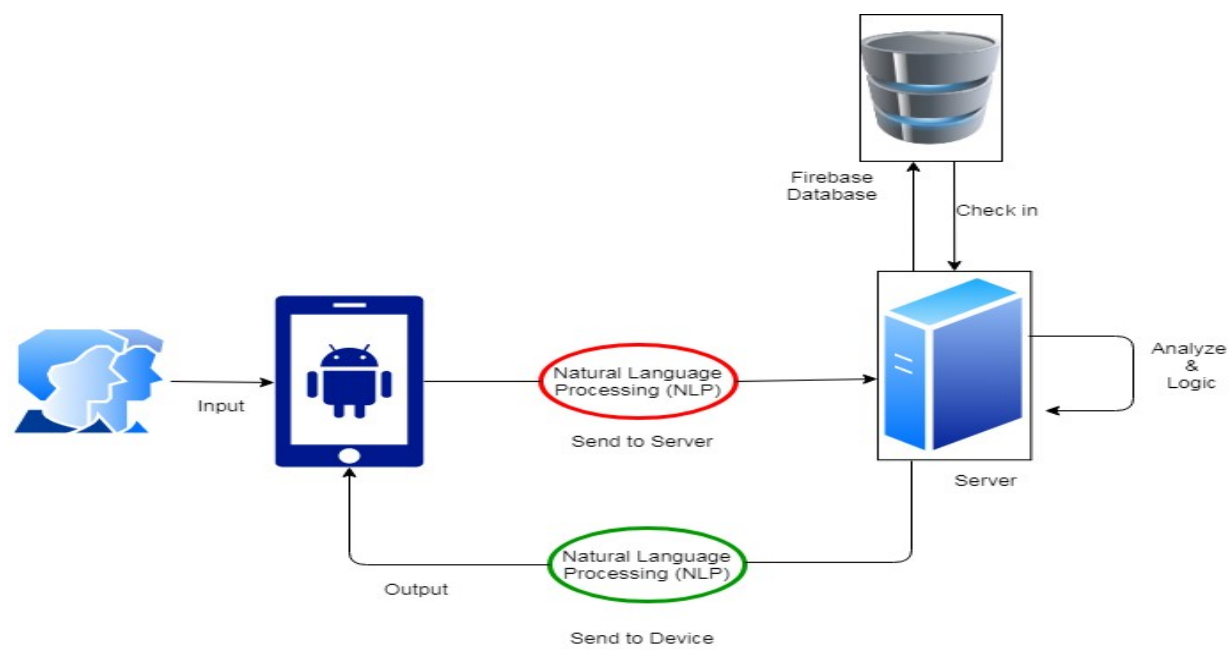

Figure 6. Second Level of System Architecture

\section{Result and Discussion}

The proposed system produces the output stating if there is Alzheimer's or not with the overall accuracy of above $95 \%$.

\subsection{Usefulness}

In this questionnaire section, four (4) item has been evaluated by the respondents for item 1 until item 4 which is about the usefulness of the application. Based on the Figure 7, the result for item 1-4, item 1 is about when the respondents were questioned that Mr. Dr. Health-assistant Chatbot application helps user be more active for healthcare. $20 \%$ respondents were strongly disagreed, and $47 \%$ respondents were agreed at this statement. While, only $33 \%$ respondents were neutral with this.

Next question for item 2 is related to statement of Mr. Dr. Health-assistant Chatbot application helps user be more productive. On this $20 \%$ respondents strongly disagreed and $53 \%$ respondents were agreed. While only $27 \%$ respondents neutral.

Another question for item 3 is related to statement of Mr. Dr. Health-assistant Chatbot application is useful to user. $13 \%$ respondents were agreed and $74 \%$ respondents were agreed with this but only $13 \%$ respondents which are neutral about that.

Next question for item 4 related to statement of Mr. Dr. Health-assistant Chatbot application gives user more control over my daily health. At this 13\% respondents were strongly disagreed and 33\% respondents were agreed with this point, but $40 \%$ respondents which were neutral about this statement. While only $13 \%$ respondents were disagree with this point. 


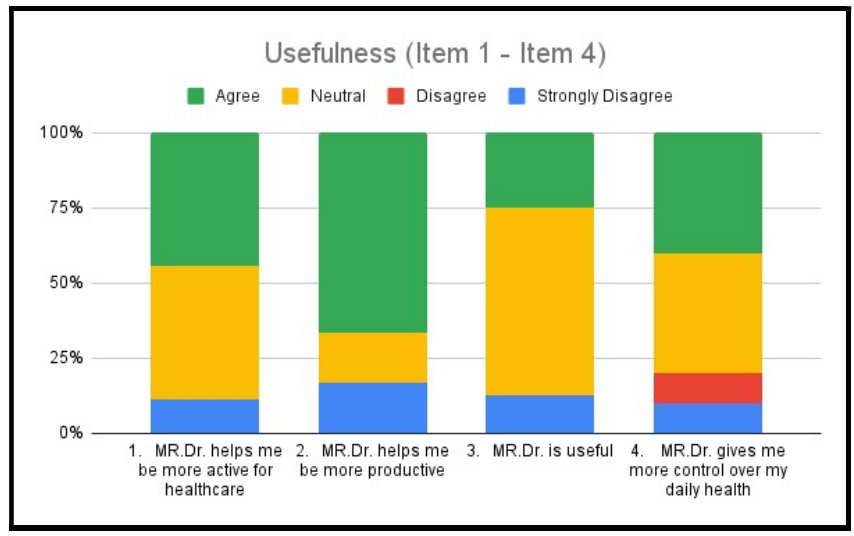

Figure 7. Usefulness

\subsection{Ease of Use}

In this questionnaire section, eleven item has been evaluated by the respondents for item 5 until item 8 which is about the ease of use of the application.

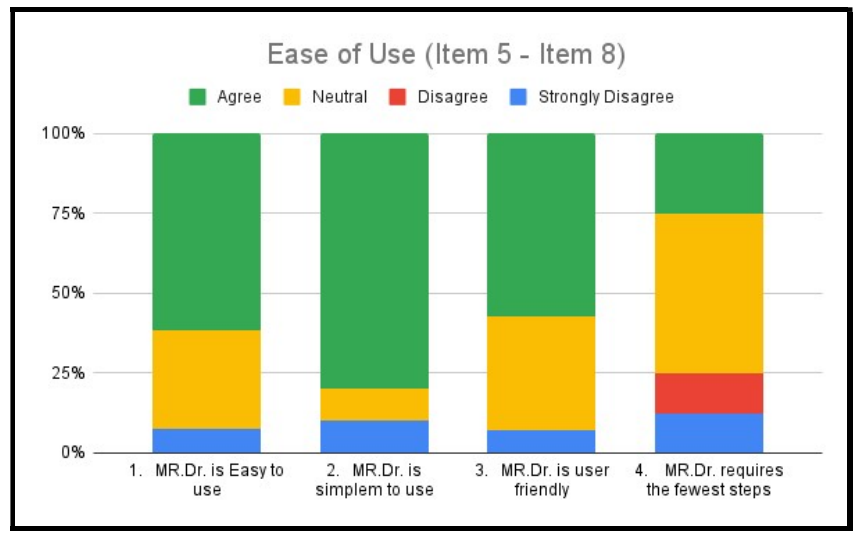

Figure 8. Ease of Use

Based on the Figure 8, the result for item 5-8, item 5 is related to the statement of Mr. Dr. Healthassistant Chatbot application is easy to use. $10 \%$ respondents were strongly agreed and $77 \%$ respondents were agreed while $13 \%$ respondents were neutral about this.

Next question for item 6 is related to the statement of Mr. Dr. Health-assistant Chatbot application is simple to use. $10 \%$ of respondents were strongly disagreed and $70 \%$ respondents were agreed. While $20 \%$ respondents were neutral with that application is simple to use.

7th item is related to the statement of Mr. Dr. Health-assistant Chatbot application is user friendly. $10 \%$ respondents were strongly agreed and $67 \%$ respondents were agreed. While $13 \%$ respondents neutral with this statement.

Next question for item 8 is related to the statement of Mr. Dr. Health-assistant Chatbot application requires the fewest steps. $13 \%$ respondents were strongly disagreed and $40 \%$ respondents were agreed while $33 \%$ respondents were neutral about this but the remaining respondents $(13 \%)$ were disagree with this statement.

\subsection{Satisfaction}

In this questionnaire section, four item has been evaluated by the respondents for item 9 until item 12 which is about satisfaction of the application. Based on the Figure 5.3, the result for item 9-12, item 9 is related to the statement of users are satisfied with Mr.Dr. Health-assistant Chatbot application. 07\% respondents were strongly disagreed and $70 \%$ respondents were agreed with this but only $13 \%$ respondents which are neutral about that. 
Next question for item 10 is related to the statement of users would recommend Mr.Dr. Healthassistant Chatbot application to a friend. In this $17 \%$ respondents were strongly disagreed while the rest of respondents $(83 \%)$ were agreed about that.

As the respondents were asked that Mr.Dr. Health-assistant Chatbot application is pleasant to use for item 11 statement, $33 \%$ respondents were strongly disagreed and 53\% respondents were agreed while $13 \%$ respondents were neutral about this.

Another question for item 12 is related to the statement of Mr.Dr. Health-assistant Chatbot application works the way users want it to work. $33 \%$ respondents were strongly disagreed and $47 \%$ respondents were agreed while $20 \%$ respondents were neutral about this statement.

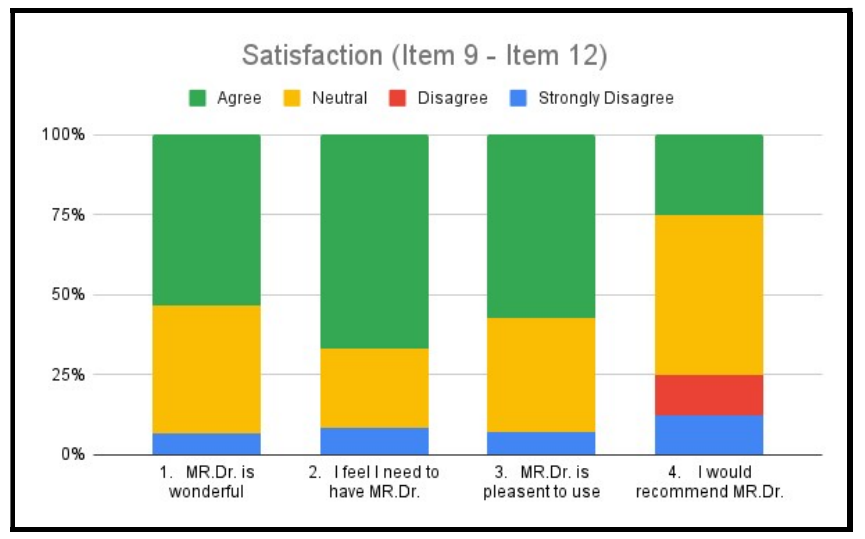

Figure 9. Satisfaction

\subsection{Analysis}

Data gathered from testing the chatbot justifies the recent growth and demand for companies wanting to integrate a chatbot. It was determined that chatbots perform at a very high standard and provide reliable and rapid responses to users compared to that of traditional methods. The average time spent interacting with the chatbot is very low as it provides an efficient way for users to get health assistant. The low interaction time reflects the high understanding and offered through the adoption of conversational user interfaces thus allowing users to freely interact with the chatbot to meet the demands of modern life. The chatbot has proven to fulfil the demand of users wanting instant access and availability information and services.

The results gathered throughout the evaluation phase support the research conducted which outlines the prevalence of chatbots within industries, particularly within the health sector such as clinic and hospital. The findings made sure that the chatbot is a very suitable method for adopting technology as a means to distribute a service. The developed chatbot allows users to interact with their clinic or hospital through natural language interaction, granting users more convenient and efficient access to their health information. This chatbot allows users to connect to various health services they may have an existing account with all from within the one communication channel through natural language.

\section{Conclusion}

The health sector industry has long been working on outdated and traditional practices. However, if we combine new technology of artificial intelligence which has lot of potential to change patients experience to communicate with the clinic and resonate with new technology. This research demonstrates that using natural language processing for building AI based conversational agent which has self-learning capabilities. The main goal of this project is to help users to ask any personal query related to healthcare without physically available to the hospital. For the recommendation of future work, this study will aim to propose an improvement of Python Ex-AR application to innovate and be responsive to enhance the learning experience of the students that can be done such as added the new contents, publish to other platform like IOS platform, plan the future commercialization and publish an application on the Google Play Store.

The chatbot could also be developed to be multilingual. Supporting multiple languages would help aid healthcare exclusion in developing countries and enhance the overall accessibility of technology 
within the banking industry whilst targeting a larger user group. From observing the users interact with the chatbot during user testing, there were numerous suggestions to integrate the chatbot across other popular platforms such as Amazon Echo or Dot, this would increase the availability of the chatbot and the integration of the DialogFlow API allow the chatbot to be easily exported Amazon Alexa.

\section{References}

[1] T.M. M. Hayir, M.A. Magan, L.M. Mohamed, M.A. Mohamud and A.A. Muse, "Barriers for full immunization coverage among under 5 years children in Mogadishu, Somalia.” Journal Family Med Prim Care, vol. 10, no. 11, pp. 2664-2673, 2020.

[2] S. Divya, V. Indumathi, S. Ishwarya, M. Priyasankari and S. K. Devi, "A self-diagnosis medical chatbot using artificial intelligence," Journal of Web Development and Web Designing, vol. 3, no. 1, pp. 1-7, 2018.

[3] U. Lanvers, "Spare a thought for the language learner!' A commentary on Hultgren's red herring," Nordic Journal of English Studies, vol. 20, no. 1, pp. 278-283, 2021 doi: $10.35360 /$ njes. 670

[4] M. Ivanovic and M. Semnic, "The Role of Agent Technologies in Personalized Medicine," in 2018 5th," International Conference on Systems and Informatics (ICSAI), 2018, IEEE. pp. 299-304, doi: 10.1109/ICSAI.2018.8599421

[5] N. Bostrom and E. Yudkowsky, "The ethics of artifificial intelligence," in Frankish $\mathrm{K}$ and Ramsey WM, The Cambridge Handbook of Artifificial Intelligence, Ed. San Cambridge: Cambridge University Press, 2014, pp. 316-334.

[6] Jiang, Fei, Yong Jiang, Hui Zhi, Yi Dong, Hao Li, Sufeng Ma, Yilong Wang, Qiang Dong, Haipeng Shen and Yongjun Wang, "Artifificial intelligence in healthcare: Past, present and future," Stroke Vasc Neurol, vol. 2, no. 4, pp. 230-243, 2017.

[7] J. He, S.L. Baxter, J. Xu, J. Xu, X. Zhou and K. Zhang, "The practical implementation of artifificial intelligence technologies in medicine," Nature Medicine, vol. 25, no. 1, pp. 30-36, 2019.

[8] D. Madhu, C. N. Jain, E. Sebastain, S. Shaji and A. Ajayakumar, "A novel approach for medical assistance using trained chatbot." In 2017 International Conference On Inventive Communication And Computational Technologies (ICICCT), 2017, pp. 243 246, IEEE.

[9] R. Dharwadkar and N. A. Deshpande, "A medical ChatBot," International Journal of Computer Trends and Technology (IJCTT), vol. 60, no. 1, pp. 41-45, 2018.

[10] V. M. Kumar, A. Keerthana, M. Madhumitha, S. Valliammai and V. Vinithasri, "Sanative chatbot for health seekers," International Journal of Engineering and Computer Science, vol. 5, no. 3, pp. 16022-16025, 2016.

[11] S. Ayanouz, B. A. Abdelhakim and M. Benhmed, "A Smart Chatbot Architecture based NLP and Machine Learning for Health Care Assistance," in Proceedings of the 3rd International Conference on Networking, Information Systems \& Security, 2020, pp. 1-6.

[12] T. Lalwani, S. Bhalotia, A. Pal, S. Bisen and V. Rathod, "Implementation of a Chat Bot System using AI and NLP," International Journal of Innovative Research in Computer Science \& Technology-IJIRCST, vol. 6, no. 3, 2018.

[13] S. Hoermann, K. L. M. Cabe, D. N. Milne and R. A. Calvo, "Application of synchronous Text-Based Dialogue Systems in Mental Health Interventions: Systematic Review," Journal of medical Internet research, vol. 19, no. 8, e267, 2017. 
[14] M. Nuruzzaman and O. K. Hussain, "A Survey on Chatbot Implementation in Customer Service Industry through Deep Neural Networks," in 2018 IEEE 15th International Conference on e-Business Engineering (ICEBE), 2018, pp. 54-61, IEEE.

[15] L. C. Klopfenstein, S. Delpriori, S. Malatini and A. Bogliolo, "The rise of bots: A survey of conversational interfaces, patterns, and paradigms," in Proceedings of the 2017 Conference on Designing Interactive systems, 2017, pp. 555-565.

[16] J. P. Campbell, "Speaker Recognition: A tutorial," Proceedings of the IEEE, vol. 85, no. 9, pp. 1437-1462, 1997.

[17] C. -H. Lee, From Knowledge-Ignorant to Knowledge-Rich Modeling: A New Speech Research Paradigm for Next Generation Automatic Speech Recognition. Canada: Springer, 2004.

[18] Bhargava, and N. Maheshwari, "An Intelligent Speech Recognition System for Education System," 2009.

[19] J. Hochfeld, N. Riffell and Levinson. "Four Trends that Willtransform Healthcarein Europe in 2016," European Pharmaceutical Review, vol. 21, no. 1, 2016.

[20] A. S. M. Mosa, I. Yoo, and L. Sheets, "A systematic Review of Healthcare Applications for Smartphones," BMC Medical Informatics and Decision Making, vol. 12, no. 1, pp. 1-31, 2012.

[21] O. Kadu, S. Sihasane, S. Naik, V. Katariya and V. S. Gutte, "Intelligent Healthbot for Transforming Healthcare," International Journal of Trend in Scientific Research and Development (IJTSRD), vol. 3, no. 3, pp. 1576-1579, 2019.

[22] D. Anas, D. Suhas, T. Manthan, and M. Harsh, "AI Based Healthcare Chatbot System Using Natural Language Processing," Thesis, St. John College of Engineering and Management, Palghar, 2019.

[23] F. Amato, S. Marrone, V. Moscato, G. Piantadosi, A. Picariello and C. Sansone, "Chatbots Meet eHealth: Automatizing Healthcare," in WAIAH@AI*IA, 2017, pp. 40-49.

[24] R. Dale, "The return of the chatbot," Natural Language Engineering, vol. 22, no. 5, pp. 811-817, 2016.

[25] S. Hudaa, D. B. P. Setiyadi, E. L. Lydia, K. Shankar, P. T. Nguyen, W. Hashim, and A. Maseleno, "Natural Language Processing Utilization in Healthcare," International Journal of Engineering and Advanced Technology,vol. 8 no. 6S2, August 2019.

[26] E. Wolfgang, "First-order predicate logic." In Introduction to Artificial Intelligence, pp. 39-64. Springer, Cham, 2017.

[27] J. P. Mueller, Beginning Programming with Python for Dummies. United States: John Wiley \& Sons 2018.

[28] L. E. Alvarez-Dionisi, M. Mittra and R. Balza, "Teaching Artificial Intelligence and Robotics to Undergraduate Systems Engineering Students," International Journal of Modern Education \& Computer Science, vol. 11, no. 7, 2019.

[29] S. Mihajlović, A. Kupusinac, D. Ivetić and I. Berković, "The Use of Python in the field of Artifical Intelligence," in International Conference on Information Technology and Development of Education - ITRO 2020, Zrenjanin, Republic of Serbia, October 2020. 